\title{
On some mathematical properties of the general zeroth-order Randić coindex of graphs
}

\author{
I. Milovanović, M. Matejić, E. Milovanović, A. Ali
}

\begin{abstract}
Let $G=(V, E), V=\left\{v_{1}, v_{2}, \ldots, v_{n}\right\}$, be a simple connected graph of order $n$, size $m$ with vertex degree sequence $\Delta=d_{1} \geq d_{2} \geq \cdots \geq d_{n}=\delta>0, d_{i}=d\left(v_{i}\right)$. Denote by $\bar{G}$ a complement of $G$. If vertices $v_{i}$ and $v_{j}$ are adjacent in $G$, we write $i \sim j$, otherwise we write $i \nsim j$. The general zeroth-order Randić coindex of $G$ is defined as ${ }^{0} \bar{R}_{\alpha}(G)=\sum_{i \nsim j}\left(d_{i}^{\alpha-1}+\right.$ $\left.d_{j}^{\alpha-1}\right)=\sum_{i=1}^{n}\left(n-1-d_{i}\right) d_{i}^{\alpha-1}$, where $\alpha$ is an arbitrary real number. Similarly, general zerothorder Randić coindex of $\bar{G}$ is defined as ${ }^{0} \bar{R}_{\alpha}(\bar{G})=\sum_{i=1}^{n} d_{i}\left(n-1-d_{i}\right)^{\alpha-1}$. New lower bounds for ${ }^{0} \bar{R}_{\alpha}(G)$ and ${ }^{0} \bar{R}_{\alpha}(\bar{G})$ are obtained. A case when $G$ has a tree structure is also covered.
\end{abstract}

Keywords: Topological indices and coindices, first Zagreb index, forgotten index, general zeroth-order Randić index.

\section{Introduction}

Let $G=(V, E), V=\left\{v_{1}, v_{2}, \ldots, v_{n}\right\}$, be a simple graph with $n \geq 3$ vertices, $m$ edges and vertex degree sequence $\Delta=d_{1} \geq d_{2} \geq \cdots \geq d_{n}=\delta>0, d_{i}=d\left(v_{i}\right)$. The complement of $G$ is the graph $\bar{G}=(V, \bar{E})$, with the same vertex set but whose edge set consists of the edges not present in $G$. Since the graph sum $G+\bar{G}$ on a $n$-node graph $G$ is the complete graph $K_{n}$, the number of edges in $\bar{G}$ is $\bar{m}=\frac{n(n-1)}{2}-m$. If vertices $v_{i}$ and $v_{j}$ are adjacent in $G$, we write $i \sim j$. On the other hand, if $v_{i}$ and $v_{j}$ are adjacent in $\bar{G}$, we write $i \nsim j$.

In graph theory, an invariant is a numerical quantity of graphs that depends only on their abstract structure, not on labeling of vertices or edges, or on the drawings of the graphs. In chemical graph theory such quantities are also referred to as topological indices [6,22-24]. Many of them are defined as simple functions of the degrees of the vertices of (molecular) graph. Most degree based topological indices are viewed as the contributions of pairs of adjacent vertices. But equally important are degree based topological indices that consider the non-adjacent pairs of vertices for computing some topological properties of graphs which are named as coindices.

Manuscript received September 21, 2020; accepted November 14, 2020.

I. Ž. Milovanović, M. M. Matejić, E. I. Milovanović are with the Faculty of Electronic Engineering, Niš, Serbia; A. Ali is with the Department of Mathematics, Faculty of Science, University of Ha'il, Ha'il, Saudi Arabia 
One of the most popular and extensively studied graph based molecular structure descriptors is the first Zagreb index introduced in 1972 by Gutman and Trinajstić in [7]. It is defined as

$$
M_{1}(G)=\sum_{i=1}^{n} d_{i}^{2}=\sum_{i \sim j}\left(d_{i}+d_{j}\right) .
$$

Various generalizations of the first Zagreb index have been proposed. In [10] a so called general zeroth-order Randić index was introduced. It was conceived as

$$
{ }^{0} R_{\alpha}(G)=\sum_{i=1}^{n} d_{i}^{\alpha}=\sum_{i \sim j}\left(d_{i}^{\alpha-1}+d_{j}^{\alpha-1}\right),
$$

where $\alpha$ is an arbitrary real number. It is also met under the names the first general Zagreb index [13] and variable first Zagreb index [17].

For specific values of $\alpha$, specific notations and hence specific names are being used. Thus, for $\alpha=2$ the aforementioned first Zagreb index is obtained. For $\alpha=3$, a so called forgotten topological index [5]

$$
F(G)=\sum_{i=1}^{n} d_{i}^{3}=\sum_{i \sim j}\left(d_{i}^{2}+d_{j}^{2}\right)
$$

is gained.

More details on these indices and their mathematical properties can be found in $[1,2,8$, $20]$ and references cited therein.

The notion of coindex was introduced in [4]. In this case sum runs over the edges of complement of $G$. The general zeroth-order Randić coindex was defined in [16] as

$$
{ }^{0} \bar{R}_{\alpha}(G)=\sum_{i \nsim j}\left(d_{i}^{\alpha-1}+d_{j}^{\alpha-1}\right)=\sum_{i=1}^{n}\left(n-1-d_{i}\right) d_{i}^{\alpha-1},
$$

where $\alpha$ is an arbitrary real number. For its mathematical properties and upper and lower bounds one can refer to $[18,19]$. For $\alpha=3$ the forgotten topological coindex, or $F$-coindex for short, is obtained [3] (see also [12]). In [25] this coindex is called the Lanzhou index.

From (1) and (2) it can be concluded that between ${ }^{0} R_{\alpha}(G)$ and ${ }^{0} \bar{R}_{\alpha}(G)$ the following relation exists [16] (see also [18])

$$
{ }^{0} R_{\alpha}(G)+{ }^{0} \bar{R}_{\alpha}(G)=(n-1){ }^{0} R_{\alpha-1}(G) .
$$

In this paper some new lower bounds for ${ }^{0} \bar{R}_{\alpha}(G)$ are obtained. A case when $G$ has a tree structure is discussed as well.

\section{Preliminaries}

In this section we recall one discrete inequality for real number sequences that will be used in proofs of theorems. 
Let $p=\left(p_{i}\right), i=1,2, \ldots, n$, be a non-negative real number sequence and $a=\left(a_{i}\right)$, $i=1,2, \ldots, n$, a sequence of positive real numbers. In [11] (see also [21]) it was proved that for any real $r, r \leq 0$ or $r \geq 1$, holds

$$
\left(\sum_{i=1}^{n} p_{i}\right)^{r-1} \sum_{i=1}^{n} p_{i} a_{i}^{r} \geq\left(\sum_{i=1}^{n} p_{i} a_{i}\right)^{r}
$$

When $0 \leq r \leq 1$, the opposite inequality is valid. Equality holds if and only if either $r=0$, or $r=1$, or $a_{1}=a_{2}=\cdots=a_{n}$, or $p_{1}=\cdots=p_{t}=0$ and $a_{t+1}=\cdots=a_{n}$, for some $t$, $1 \leq t \leq n-1$.

\section{Main results}

In the next theorem we establish a connection between ${ }^{0} \bar{R}_{\alpha}(G)$ and $M_{1}(G)$.

Theorem 3.1. Let $G, G \nsucceq K_{n}$, be a simple connected graph with $n \geq 3$ vertices and $m$ edges. Then, for any real $\alpha, \alpha \leq 1$ or $\alpha \geq 2$, holds

$$
{ }^{0} \bar{R}_{\alpha}(G) \geq(n-1)\left(\delta^{\alpha-1}+\Delta^{\alpha-1}\right)-\delta^{\alpha}-\Delta^{\alpha}+\frac{\left((n-1)(2 m-\delta-\Delta)-M_{1}(G)+\delta^{2}+\Delta^{2}\right)^{\alpha-1}}{((n-1)(n-2)-2 m+\delta+\Delta)^{\alpha-2}} .
$$

When $1 \leq \alpha \leq 2$ the opposite inequality is valid. Equality holds if and only if either $\alpha=1$, or $\alpha=2$, or $n-1=\Delta=d_{1}=\cdots=d_{t}>d_{t+1}=\cdots=d_{n-1} \geq d_{n}=\delta$, for some $t$, $1 \leq t \leq n-2$, or $n-1 \neq \Delta=d_{1}=d_{2}=\cdots=d_{n-1} \geq d_{n}=\delta$.

Proof. The inequality (3) can be observed in the form

$$
\left(\sum_{i=2}^{n-1} p_{i}\right)^{r-1} \sum_{i=2}^{n-1} p_{i} a_{i}^{r} \geq\left(\sum_{i=2}^{n-1} p_{i} a_{i}\right)^{r}
$$

For $r=\alpha-1, \alpha \leq 1$ or $\alpha \geq 2, p_{i}=n-1-d_{i}, a_{i}=d_{i}, i=1,2, \ldots, n$, the above inequality becomes

$$
\left(\sum_{i=2}^{n-1}\left(n-1-d_{i}\right)\right)^{\alpha-2} \sum_{i=2}^{n-1}\left(n-1-d_{i}\right) d_{i}^{\alpha-1} \geq\left(\sum_{i=2}^{n-1}\left(n-1-d_{i}\right) d_{i}\right)^{\alpha-1} .
$$

If $d_{2}=\cdots=d_{n-1}=n-1$, that is if $G \cong K_{n}$, in (6) equality occurs. Since ${ }^{0} \bar{R}_{\alpha}\left(K_{n}\right)=0$, without affecting the generality, suppose that $G \nsucceq K_{n}$. Then, according to (6) we have that

$$
\sum_{i=2}^{n-1}\left(n-1-d_{i}\right) d_{i}^{\alpha-1} \geq \frac{\left(\sum_{i=2}^{n-1}\left(n-1-d_{i}\right) d_{i}\right)^{\alpha-1}}{\left(\sum_{i=2}^{n-1}\left(n-1-d_{i}\right)\right)^{\alpha-2}}
$$

from which (4) is obtained.

The case when $1 \leq \alpha \leq 2$ is proved analogously.

Equality in (7), and consequently in (4), holds if and only if either $\alpha=1$, or $\alpha=2$, or $n-1=\Delta=d_{1}=\cdots=d_{t}>d_{t+1}=\cdots=d_{n-1} \geq d_{n}=\delta$, for some $t, 1 \leq t \leq n-2$, or $n-1 \neq \Delta=d_{1} \geq d_{2}=\cdots=d_{n-1} \geq d_{n}=\delta$. 
Corollary 3.1. Let $G, G \nsubseteq K_{n}$, be a simple connected graph with $n \geq 3$ vertices and $m$ edges. Then

$$
\bar{F}(G) \geq(n-1)\left(\delta^{2}+\Delta^{2}\right)-\delta^{3}-\Delta^{3}+\frac{\left((n-1)(2 m-\Delta-\delta)-M_{1}(G)+\delta^{2}+\Delta^{2}\right)^{2}}{(n-1)(n-2)-2 m+\Delta+\delta} .
$$

Equality holds if and only if $n-1=\Delta=d_{1}=\cdots=d_{t}>d_{t+1}=\cdots=d_{n-1} \geq d_{n}=\delta$, for some $t, 1 \leq t \leq n-2$, or $n-1 \neq \Delta=d_{1} \geq d_{2}=\cdots=d_{n-1} \geq d_{n}=\delta$.

In the next theorem we determine a lower bound on ${ }^{0} \bar{R}_{\alpha}(G)$ when $G$ is a tree, $G \cong T$.

Theorem 3.2. Let $T$ be a tree with $n \geq 4$ vertices. Then, for any real $\alpha, \alpha \leq 1$ or $\alpha \geq 2$, holds

$$
{ }^{0} \bar{R}_{\alpha}(T) \geq 2(n-2)+\frac{\left(2\left(n^{2}-3 n+3\right)-M_{1}(T)\right)^{\alpha-1}}{(n-2)^{\alpha-2}(n-3)^{\alpha-2}} .
$$

When $1 \leq \alpha \leq 2$, the opposite inequality is valid. Equality holds if and only if either $\alpha=1$, or $\alpha=2$, or $T \cong P_{n}$ or $T \cong K_{1, n-1}$.

Proof. The inequality (3) can be considered as

$$
\left(\sum_{i=1}^{n-2} p_{i}\right)^{r-1} \sum_{i=1}^{n-2} p_{i} a_{i}^{r} \geq\left(\sum_{i=1}^{n-2} p_{i} a_{i}\right)^{r}
$$

For $r=\alpha-1, \alpha \leq 1$ or $\alpha \geq 2, p_{i}=n-1-d_{i}, a_{i}=d_{i}, i=1,2, \ldots, n$, the above inequality transforms into

$$
\left(\sum_{i=1}^{n-2}\left(n-1-d_{i}\right)\right)^{\alpha-2} \sum_{i=1}^{n-2}\left(n-1-d_{i}\right) d_{i}^{\alpha-1} \geq\left(\sum_{i=1}^{n-2}\left(n-1-d_{i}\right) d_{i}\right)^{\alpha-1}
$$

Let $G$ be a tree with $n \geq 4$ vertices. Then, $m=n-1$ and $d_{n-1}=d_{n}=\Delta=1$, since any tree has at least two vertices of degree 1 . Now, the inequality (10) becomes

$$
\begin{aligned}
& (n(n-1)-2(n-1)-2(n-2))^{\alpha-2}\left(\sum_{i=1}^{n}\left(n-1-d_{i}\right) d_{i}^{\alpha-1}-2(n-2)\right) \geq \\
& \geq\left(2(n-1)^{2}-M_{1}(T)-2(n-2)\right)^{\alpha-1},
\end{aligned}
$$

that is

$$
(n-2)^{\alpha-2}(n-3)^{\alpha-2}\left({ }^{0} \bar{R}_{\alpha}(T)-2(n-2)\right) \geq\left(2\left(n^{2}-3 n+3\right)-M_{1}(T)\right)^{\alpha-1},
$$

from which (8) is obtained.

Equality in (11) holds if and only if either $\alpha=1$, or $\alpha=2$, or $n-1=\Delta=d_{1}=\cdots=$ $d_{t}>d_{t+1}=\cdots=d_{n-2} \geq d_{n-1}=d_{n}=\delta=1$, for some $t, 1 \leq t \leq n-3$, or $n-1 \neq \Delta=$ $d_{1}=\cdots=d_{n-2} \geq d_{n-1}=d_{n}=\delta=1$, which implies that equality in (8) holds if and only if either $\alpha=1$, or $\alpha=2$, or $T \cong P_{n}$, or $T \cong K_{1, n-1}$. 
Corollary 3.2. Let $T$ be a tree with $n \geq 4$ vertices. Then

$$
\bar{F}(T) \geq 2(n-2)+\frac{\left(2\left(n^{2}-3 n+3\right)-M_{1}(T)\right)^{2}}{(n-2)(n-3)} .
$$

Equality holds if and only if $T \cong P_{n}$ or $T \cong K_{1, n-1}$.

Corollary 3.3. Let $T$ be a tree with $n \geq 2$ vertices. Then

$$
\bar{F}(T) \geq(n-2)(n-1) .
$$

Equality holds if and only if $T \cong K_{1, n-1}$.

Proof. In [9] (see also [14]) the following inequality was proven

$$
M_{1}(T) \leq n(n-1),
$$

with equality if and only if $T=K_{1, n-1}$. From this and (12), the inequality (13) is obtained.

The inequality (13) was proven in [25].

Denote by $\Gamma_{1}$ a class of connected graphs that do not have vertices of degree $n-1$. In the next theorem we determine a lower bound for ${ }^{0} \bar{R}_{\alpha}(\bar{G})$ in terms of $M_{1}(G)$ and $n, m, \delta$ and $\Delta$.

Theorem 3.3. Let $G$ be a simple connected graph with $n \geq 3$ vertices and $m$ edges. Then, for any real $\alpha, \alpha \leq 1$ or $\alpha \geq 2$, holds

$$
\begin{aligned}
{ }^{0} \bar{R}_{\alpha}(\bar{G}) & \geq \Delta(n-1-\Delta)^{\alpha-1}+ \\
& +\delta(n-1-\delta)^{\alpha-1}+\frac{\left((n-1)(2 m-\Delta-\delta)-M_{1}(G)+\delta^{2}+\Delta^{2}\right)^{\alpha-1}}{(2 m-\Delta-\delta)^{\alpha-2}}
\end{aligned}
$$

When $1 \leq \alpha \leq 2$, the opposite inequality is valid.

If $G$ belong to the class $\Gamma_{1}$, then for any real $\alpha \leq 1$ inequality (14) is valid.

Equality holds if and only if either $\alpha=1$, or $\alpha=2$, or $\Delta=d_{1} \geq d_{2}=\cdots=d_{n-1} \geq d_{n}=$ $\delta$ and $\alpha \geq 1$, or $n-1 \neq \Delta=d_{1} \geq d_{2}=\cdots=d_{n-1} \geq d_{n}=\delta$ and $\alpha \leq 1$.

Proof. For $r=\alpha-1, \alpha \geq 2, p_{i}=d_{i}, a_{i}=n-1-d_{i}, i=1,2, \ldots, n$, the inequality (5) becomes

$$
\left(\sum_{i=2}^{n-1} d_{i}\right)^{\alpha-2} \sum_{i=2}^{n-1} d_{i}\left(n-1-d_{i}\right)^{\alpha-1} \geq\left(\sum_{i=2}^{n-1} d_{i}\left(n-1-d_{i}\right)\right)^{\alpha-1}
$$

that is

$$
\begin{aligned}
{ }^{0} \bar{R}_{\alpha}(\bar{G})-\Delta(n-1-\Delta)^{\alpha-1}-\delta(n-1-\delta)^{\alpha-1} \geq \\
\geq \frac{\left(2 m(n-1)-M_{1}(G)-\Delta(n-1-\Delta)-\delta(n-1-\Delta)\right)^{\alpha-1}}{(2 m-\Delta-\delta)^{\alpha-2}}
\end{aligned}
$$


from which (14) is obtained.

The case when $1 \leq \alpha \leq 2$ is proved analogously. Similarly, it can be proved that when $\alpha \leq 1$ and $G$ does not belong to class $\Gamma_{1}$ the inequality (14) is valid.

Equality in (15) holds if and only if either $\alpha=1$, or $\alpha=2$, or $n-1-d_{2}=n-1-d_{3}=$ $\cdots=n-1-d_{n-1}$, which implies that equality in (14) holds if and only if either $\alpha=1$, or $\alpha=2$, or $\Delta=d_{1} \geq d_{2}=\cdots=d_{n-1} \geq d_{n}=\delta$ and $\alpha=1$, or $n-1 \neq \Delta=d_{1} \geq d_{2}=\cdots=$ $d_{n-1} \geq d_{n}=\delta$ and $\alpha \leq 1$..

Corollary 3.4. Let $G$ be a simple connected graph with $n \geq 3$ vertices and $m$ edges. Then

$$
\bar{F}(\bar{G}) \geq \Delta(n-1-\Delta)^{2}+\delta(n-1-\delta)^{2}+\frac{\left((n-1)(2 m-\Delta-\delta)-M_{1}(G)+\delta^{2}+\Delta^{2}\right)^{2}}{2 m-\Delta-\delta} .
$$

Equality holds if and only if $\Delta=d_{1} \geq d_{2}=\cdots=d_{n-1} \geq d_{n}=\delta$.

The proof of the next theorem is similar to that of Theorem 3.2.

Theorem 3.4. Let $T$ be a tree with $n \geq 3$ vertices. Then, for any real $\alpha \geq 2$, holds

$$
{ }^{0} \bar{R}_{\alpha}(\bar{T}) \geq 2(n-2)^{\alpha-2}+\frac{\left(2\left(n^{2}-3 n+3\right)-M_{1}(T)\right)^{\alpha-1}}{(2(n-2))^{\alpha-2}} .
$$

When $1 \leq \alpha \leq 2$, the opposite inequality is valid. When $\alpha \leq 1$ and $T ¥ K_{1, n-1}$, the inequality (16) is also valid.

Equality holds if and only if either $\alpha=1$, or $\alpha=2$, or $T \cong P_{n}$.

Corollary 3.5. Let $T$ be a tree with $n \geq 3$ vertices. Then

$$
\bar{F}(\bar{T}) \geq 2(n-2)+\frac{\left(2\left(n^{2}-3 n+3\right)-M_{1}(T)\right)^{2}}{2(n-2)} .
$$

Equality holds if and only if $T \cong P_{n}$.

From (8) and (16) we have that the following is valid:

Corollary 3.6. Let $T$ be a tree with $n \geq 4$ vertices. Then, for any real $\alpha \geq 2$, holds

$$
\begin{aligned}
{ }^{0} \bar{R}_{\alpha}(T) & +{ }^{0} \bar{R}_{\alpha}(\bar{T}) \geq 2(n-2)\left(1+(n-2)^{\alpha-3}\right)+ \\
+ & \frac{\left(2\left(n^{2}-3 n+3\right)-M_{1}(T)\right)^{\alpha-1}\left(2^{\alpha-2}+(n-3)^{\alpha-2}\right)}{2^{\alpha-2}(n-2)^{\alpha-2}(n-3)^{\alpha-2}} .
\end{aligned}
$$

The inequality (18) is also valid when $\alpha \leq 1$ and $T \not K_{1, n-1}$. When $1 \leq \alpha \leq 2$ the opposite inequality in (18) is valid. Equality holds if and only if either $\alpha=1$, or $\alpha=2$, or $T \cong P_{n}$.

Corollary 3.7. Let $T$ be a tree with $n \geq 4$ vertices. Then

$$
\bar{F}(T)+\bar{F}(\bar{T}) \geq 4(n-2)+\frac{(n-1)\left(2\left(n^{2}-3 n+3\right)-M_{1}(T)\right)^{2}}{2(n-2)(n-3)} .
$$

Equality holds if and only if $T \cong P_{n}$. 
On some mathematical properties of the general zeroth-order Randić coindex of graphs

\section{References}

[1] A. Ali, I. Gutman, E. Milovanović, I. Milovanović, Sum of powers of the degrees of graphs: extremal results and bounds, MATCH Commun. Math. Comput. Chem. 80 (2018) 5-84.

[2] B. Borovičanin, K. C. Das, B. Furtula, I. Gutman, Bounds for Zagreb indices, MATCH Commun. Math. Comput. Chem. 78 (2017) 17-100.

[3] N. De, S. M. A. NAYEem, A. PAL, The F-coindex of some graph operations, SpringerPlus 5 (2016) \#221.

[4] T. DošLić, Vertex-weighted Wiener polynomials for composite graphs, Ars Math. Comp. 1 (2008) 66-80.

[5] B. Furtula, I. Gutman, A forgotten topological index, J. Math. Chem. 53 (4) (2015) 11841190.

[6] I. Gutman, Degree-based topological indices, Croat. Chem. Acta 86 (2013) 351-361.

[7] I. Gutman, N. TRinajstić, Graph theory and molecular orbitals. Total $\pi$-electron energy of alternant hydrocarbons, Chem. Phys. Lett. 17 (1972) 535-538.

[8] I. Gutman, E. Milovanović, I. Milovanović, Beyond the Zagreb indices, AKCE Int. J. Graph. Comb. 17 (2020) 74-85.

[9] I. Gutman, K. C. DAs, The first Zagreb index 30 years after, MATCH Commun. Math. Comput. Chem. 50 (2004) 83-92.

[10] Y. HU, X. Li, T. XU, I. GUTMAn, On molecular graphs with smallest and greatest zerothorder Randić index, MATCH Commun. Math. Comput. Chem. 54 (2005) 425-434.

[11] J. L. W. JENSEN, Sur les functions convexes of les integralities entre les valuesmoyennes, Acta Math. 30 (1906) 175-193.

[12] A. Khaksari, M. Chorbani, On the forgotten topological index, J. Math. Chem. 8 (2017) 327-338.

[13] X. LI, J. ZHENG, A unified approach to the extremal trees for different indices, MATCH Commun. Math. Comput. Chem. 54 (2005) 195-208.

[14] X. LI, H. ZHAO, Trees with the first three smallest and largest generalized topological indices, MATCH Commun. Math. Comput. Chem. 50 (2004) 57-62.

[15] J. B. LiU, M. M. Matejić, E. I. Milovanović, I. Ž. Milovanović, Some new inequalities for the forgotten index and coindex of graphs, MATCH Commun. Math. Comput. Chem. 84 (2020) 719-738.

[16] T. MAnsour, C. Song, The a and $(a, b)$-analogs of Zagreb indices and coindices of graphs, Int. J. Comb. 2012 (2012) Art. ID 909285.

[17] A. Miličević, S. Nikolić, On variable Zagreb indices, Croat. Chem. Acta 77 (2004) 97101.

[18] I. Milovanović, E. Milovanović, M. Matejić, Some remarks on the general zerothorder Randić coindex, Filomat (in press).

[19] I. Milovanović, M. Matejić, E. Milovanović, A note on the zeroth-order Randić coindex of graphs, Contrib. Math. 1 (2020) 17-21.

[20] P. Milošević, I. Milovanović, E. Milovanović, M. Matejić, Some inequalities for general zeroth-order Randić index, Filomat 33 (2019) 5251-5260. 
[21] D. S. Mitrinović, J. E. PeČArić, A. M. Fink, Classical and new inequalities in analysis, Kluwer Academic Publishers, Dorchrecht, 1993.

[22] R. Todeschini, V. Consonni, Handbook of molecular descriptors, Wiley VCH, Weinheim, 2000.

[23] R. Todeschini, V. Consonni, New local vetrex invariants and molecular descriptors based on the vertex degrees, MATCH Commun. Math. Comput. Chem. 64 (2010) 359-372.

[24] B. VuKIČEVIĆ, Bond additive modeling 2. Mathematical properties of maximum radeg index, Croat. Chem. Acta 83 (2010) 261-273.

[25] D. Vukičević, Q. Li, J. Sedlar, T. Došlić, Lanzhou index, MATCH Commun. Math. Comput. Chem. 80 (2018) 863-876. 